\title{
PROPOSTA DE MELHORIA NO PROCESSO DE COMBATE À CRIMINALIDADE NA CIDADE DO RIO DE JANEIRO
}

\author{
Marcone Freitas dos Reis \\ Centro de Tecnologia da Indústria Química e Têxtil (SENAI CETIQT) \\ Rua Magalhães Castro, 174 - Riachuelo, Rio de Janeiro - RJ, 20961-020 \\ marconefreis11@gmail.com \\ Vitor Ribeiro Cuervo \\ Centro de Tecnologia da Indústria Química e Têxtil (SENAI CETIQT) \\ Rua Magalhães Castro, 174 - Riachuelo, Rio de Janeiro - RJ, 20961-020 \\ vitor.cuervo@hotmail.com
}

Marcos dos Santos

Instituto Militar de Engenharia (IME)

Praça General Tibúrcio, 80 - Praia Vermelha - Urca, Rio de Janeiro - RJ, 22290-270

marcosdossantos_doutorado_uff@yahoo.com.br

Angélica Rodrigues de Lima

Centro de Tecnologia da Indústria Química e Têxtil (SENAI CETIQT)

Rua Magalhães Castro, 174 - Riachuelo, Rio de Janeiro - RJ, 20961-020

angelicadelima.engprod@gmail.com

\section{Ernesto Rademaker Martins}

Centro de Análise de Sistemas Navais (CASNAV)

Praça Barão de Ladário s/n - Ilha das Cobras - Rio de Janeiro - RJ - CEP: 20091-000

radmart@yahoo.com.br

\section{RESUMO}

Os índices de criminalidade nos grandes centros urbanos têm se intensificado nos últimos anos, devido ao aumento populacional e a falta de infraestrutura nas cidades. Em paralelo, a tecnologia de informação, dispositivos móveis e sensores alavancam um novo paradigma aonde vem sendo gerado um grande volume de dados. Tendo em vista que há um avanço tímido nas práticas de integração da gestão pública, não raros tomadas de decisão acontecem de forma desarticulada e imprecisa. O objetivo do presente trabalho é propor melhorias no processo de combate à criminalidade na cidade do Rio de Janeiro, sugerindo a utilização do Big Data para coletar os dados, e onde esse grande volume de dados dá base às tomadas de decisão integradas dos setores da gestão municipal. O método utilizado para a pesquisa envolveu o estudo teórico sobre o tema e o levantamento de exemplos de utilização do Big Data. A partir do levantamento dos índices de criminalidade no bairro da Tijuca situado na zona norte da cidade do Rio de Janeiro, é apresentado uma proposta de melhoria no combate à criminalidade para o auxílio da gestão pública integrada e base às tomadas de decisão.

Palavra-chave: Segurança Pública; Criminalidade; Big Data; Inteligência Artificial. 


\begin{abstract}
Crime rates in large urban centers have intensified in recent years due to population growth and lack of infrastructure in cities. In parallel, information technology, mobile devices and sensors are leveraging a new paradigm where a large volume of data has been generated. Given that there is a timid advance in public management integration practices, decisions often take place in an inarticulate and imprecise manner. The objective of this paper is to propose improvements in the process of combating crime in the city of Rio de Janeiro, suggesting the use of Big Data to collect data, and where this large volume of data underpins the integrated decision making of the management sectors. municipal. The method used for the research involved the theoretical study on the subject and the survey of examples of use of Big Data. From the survey of crime rates in the Tijuca neighborhood in the north of the city of Rio de Janeiro, it is presented a proposal to improve the fight against crime to help the integrated public management and basis for decision making.
\end{abstract}

Keywords: Public Security; Crime; Big Data; Artificial Intelligence.

\title{
Como Citar:
}

REIS, Marcone Freitas dos; CUERVO, Vitor Ribeiro; SANTOS, Marcos dos; LIMA, Angélica Rodrigues de; MARTINS, Ernesto Rademaker. Proposta de Melhoria no Processo de Combate à Criminalidade na Cidade do Rio de Janeiro. In: SIMPÓSIO DE PESQUISA OPERACIONAL E LOGÍSTICA DA MARINHA, 19., 2019, Rio de Janeiro, RJ. Anais [...]. Rio de Janeiro: Centro de Análises de Sistemas Navais, 2019.

\section{INTRODUÇÃO}

A cada dia que passa as pessoas buscam respostas mais rápidas e mais objetivas para certas pesquisas e para que possamos ter esse objetivo alcançado nós precisamos conhecê-las o máximo possível.

De acordo com Beath et al. (2012), devido ao crescimento de dados não estruturados e ao valor da informação, que vem sendo identificados e explorados pelas organizações, esse tem sido um tema que vem criando muito interesse. "Tomada de decisão baseada em evidências (também conhecido como big data) não é apenas a última moda, é o futuro de como vamos orientar e fazer crescer o negócio”. (HAMMOND, 2013)

Segundo Choo (2006), para ser obter uma decisão racional, deve se listar todas as informações existentes e conseguir identificar seus resultados. Para isso ocorrer, é preciso obter todas as informações essenciais. (GOMES, 2007)

Para Mcafee e Brynjolfsson (2012), a quantidade de informações só vem crescendo cada vez mais. Em 2012, aproximadamente 2,4 exabytes de dados foram criados diariamente. A previsão é que para daqui a 2 anos, será produzido mais de 40.000 exabytes, o que daria cerca de mais de 5.200 gigabytes para cada habitante. (GRANTZ E REINSEL, 2012)

Davenport et al. (2012) diz que, o maior problema das próximas décadas para as organizações é saber utilizar desses dados de forma inteligente para auxiliar na tomada de decisão, sejam eles estruturados ou não estruturados.

Segundo Galbraith (2014), com a facilidade e velocidade de geração de dados e trocas de informações decorrente a criação da internet, junto com a grande capacidade de armazenamento que se têm hoje, esses dados estão sendo extraídos de diversas fontes, como fotos de celulares, GPS (Global Positioning System), áudio de call centers, e-mails, redes 
sociais e blogs, de forma instantânea.

Harriott (2013) corrobora que, a dificuldade é em selecionar os dados que possam agregar informações pertinentes, dentro de tantas fontes possíveis e extrair a melhor informação que interprete esses dados.

Para o presente estudo, será sugerido a utilização do big data para capturar o máximo de informações possíveis e essas informações possibilitar a tomada de decisões para combate à violência na cidade do Rio de Janeiro.

\section{PROBLEMA}

A violência vem aumentando no Rio de Janeiro, através de dados do ISP (Instituto de Segurança Pública), pode se observar que em 2017 para cada 100 mil habitantes o estado teve 40 mortes violentas, porém este não foi o único indicador negativo, os principais tipos de roubos - cargas, veículos, celulares e em coletivos - tiveram altas históricas, aumentando a insegurança da população.

Os roubos de cargas que acarretaram um prejuízo de R\$ 607,1 milhões em 2017 alcançaram a marca de 10.599 casos, refere se a 304\% maior ao registrado em 2010. Já os de veículos que tiveram 54.367 casos em 2017, obtiveram um percentual de 189,6\% maior que 2010, onde foram registrados 18.773 casos. Já os assaltos em coletivos saltaram de 8.202 em 2010 para 15.283 em 2017, um aumento de 86,3\%. Já os roubos de celulares chegaram a 24.387 casos, alta de 284,9\% em comparação aos 6.988 registrados em 2010.

O estado do Rio de Janeiro em 2017 registrou 230.450 roubos, isso significa que a cada 2 minutos ocorrem um caso. Levando se em consideração todas as modalidades de crime, representa um recorde, desde que o ISP começou a controlar o índice de crimes em 1991. A Zona Norte da capital foi à região que mais sofreu com esse aumento, onde sete das dez delegacias que estão entre as que mais registram crimes, são dessa região.

A Figura 1 a seguir, apresenta as 20 áreas mais críticas na cidade do Rio de Janeiro.

Figura 1 - As vinte áreas mais críticas - Total de Roubos

\begin{tabular}{|c|c|c|c|}
\hline Delegacia & 2016 & 2017 & VARIAÇĀO, EM\% \\
\hline $26^{\mathrm{a}}$ Todos os Santos & 1.790 & 3.163 & 76,7 \\
\hline $25^{a}$ Engenho Novo & 2.543 & 4.000 & 57,3 \\
\hline $19^{a}$ Tijuca & 1.512 & 2.313 & 53,0 \\
\hline $29^{a}$ Madureira & 4.606 & 6.881 & 49,4 \\
\hline $18^{\mathrm{a}}$ Praça da Bandeira & 2.347 & 3.320 & 41,5 \\
\hline $17^{\text {a }}$ Sao Cristordo & 2.523 & 3.544 & 40,5 \\
\hline $37^{\mathrm{a}}$ tha do Govemador & 1.242 & 1.703 & 37,1 \\
\hline $7^{\mathrm{a}} \quad$ Santa Teresa & 843 & 1.154 & 36,9 \\
\hline $10^{a}$ Botafogo & 1.264 & 1.715 & 35,7 \\
\hline $32^{\mathrm{a}}$ Taquara & 2.302 & 3.110 & 35,1 \\
\hline $9^{a}$ Caltete & 1.468 & 1.983 & 35,1 \\
\hline $16^{a}$ Barra da Tivuca & 1.943 & 2.544 & 30,9 \\
\hline $15^{a}$ Gáves & 435 & 566 & 30,1 \\
\hline $30^{a}$ Marechal Hermes & 2.400 & 3.067 & 27,8 \\
\hline $24^{a}$ Piedade & 2.247 & 2.847 & 26,7 \\
\hline $42^{\mathrm{a}}$ Recrevio & 1.256 & 1.588 & 26,4 \\
\hline $20^{a}$ Via isabel & 2.100 & 2.653 & 26,3 \\
\hline $40^{\mathrm{a}}$ Honotio Gurgel & 5.079 & 6.383 & 25,7 \\
\hline $36^{\mathrm{a}}$ Santa Cruz & 1.803 & 2.253 & 25,0 \\
\hline $34^{\mathrm{a}}$ Bangu & 6.645 & 8.266 & 24,4 \\
\hline
\end{tabular}

Fonte: Jornal Extra (2018) 
Na Figura 2 a seguir, é apresentado o mapa mental elaborado para facilitar o entendimento da proposta de melhorias no processo de combate à criminalidade utilizando a ferramenta big data para auxiliar na tomada de decisão.

Figura 2 - Mapa mental

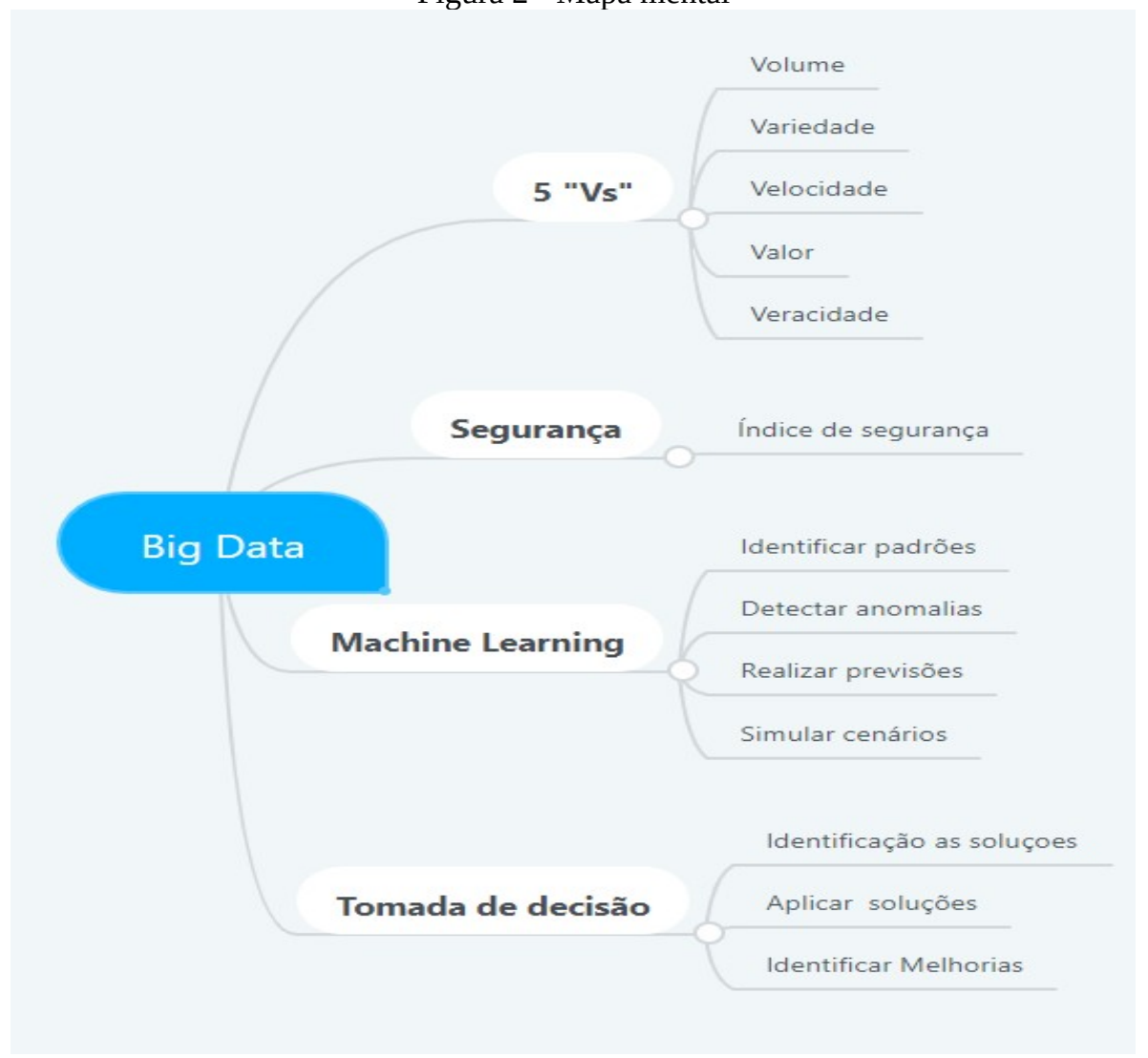

Fonte: Autores (2018)

\section{FUNDAMENTAÇÃO TEÓRICA}

\subsection{BIG DATA}

Embora o termo big data tenha surgido nos anos 90, começou a ser desenvolvido e ser utilizados por empresas nos últimos anos.

O big data tem como característica principal o chamado 5 "Vs", que são elas: volume, velocidade, variedade, veracidade e valor. Através dessas características principais, podemos aplicar o big data em diversas áreas.

Big data é o conjunto de dados do qual o tamanho é além da capacidade de ferramentas de software de banco de dados tradicionais, típicos para capturar, armazenar, gerenciar e analisar. (MANYIKA, 2011)

Já Mcafee (2012), pensa no big data como uma fundamental maneira para aperfeiçoar a eficiência e a eficácia das organizações de vendas e marketing. Ao empregar o uso do big data no coração de vendas e marketing, os insights podem ser utilizados para melhorar a tomada de decisão e inovar no modelo de vendas da empresa, podendo envolver a utilização desses dados para orientar certas ações em tempo real.

Segundo Demirkan (2012), existe o desafio de administrar essa grande quantidade de dados que está ficando cada vez maiores por causa do armazenamento barato e da 
evolução dos dados cada vez mais digitais através dos dispositivos de coleta de informações, como celulares, laptops entre outros.

O fenômeno surgiu nos últimos anos graças à enorme quantidade de dados que vem sendo gerada. Simultaneamente as informações adicionais obtidas por análise de todas essas informações, que por si só cria outro conjunto de dados enormes. (PHELAN, 2012)

Para Savitz (2012), big data é a um conjunto de grandes dados e complexos, de forma que é impossível processá-los em ferramentas usuais. Para o autor, big data é difícil de capturar, armazenar, pesquisar, compartilhar, analisar e visualizar.

\subsection{Os 5 "Vs"}

\subsubsection{Volume}

Segundo Tankard (2012), gerar e armazenar esse grande volume de dados com as ferramentas tradicionais é um grande desafio.

As variáveis envolvidas são:

- Volume de informações;

- Acessibilidade de informações;

- Mudança do comportamento em função do volume.

\subsubsection{Variedade}

McAfee e Brynjolfsson (2012) e Preimesberger (2011) apontam a variedade de dados através de e-mails, registros de cartões de crédito e de sensores, informações de redes sociais, assim como fotos e arquivos audiovisuais e gráficos.

Variáveis envolvidas:

- Variedade de fontes de informação;

- Variedade de tipos de dados.

\subsubsection{Velocidade}

McAfee e Brynjolfsson (2012) e Preimesberger (2011), utilizam o argumento de que é preciso saber trabalhar com a velocidade, pois pode acabar inviabilizando uma operação, pois a própria pode ser um limitador da análise, caso um software não seja carregado em tempo real, quando a velocidade dos dados é esta. Segundo Taurion (2012) muitas vezes precisamos conduzir em tempo real, reivindicando assim um processamento que acompanhe essa velocidade.

Variáveis envolvidas:

- Velocidade de chegada da informação;

- Velocidade da tomada de decisão.

\subsubsection{Valor}

Maniyka et al. (2011), ressalta a confiança e a experiência para a retirada de valor na análise rigorosa de dados do big data. Tankard (2012) realça a clareza como uma importante característica para atribuir valor as informações.

Variáveis envolvidas:

- Resultado agregado a partir das análises das informações;

- Qualidade das informações;

- Valor financeiro para ter dados de qualidade.

\subsubsection{Veracidade}

Para Taurion (2012), é indispensável “ter certeza que os dados fazem sentido e são autênticos”. Para a informação verdadeira tem afinidade com informações exatas, 
consistentes e relevantes, características da qualidade da informação, podendo assim ser utilizadas como bases por gestores para conseguir responder aos desafios estratégicos e operacionais. (WEBER et. al., 2009)

Variáveis envolvidas:

- Qualidade da informação

\subsection{SEgurança PÚBlica}

Segundo Arthur Trindade (2017), a violência no Rio de Janeiro apresenta características que não são encontradas em outros locais do país, no Rio de Janeiro, é o único estado que a polícia precisa de caveirões (veículos blindados) para usar em operações onde o tráfico está presente. Além disso, qualquer troca de tiros no Rio de Janeiro tem uso de fuzil por partes dos traficantes, o que é bem mais difícil de ver em outros estados.

Ignacio Cano (2017) cita que o número de roubos começou a crescer em 2013 e, desde então, aumenta aceleradamente. Para Ignacio, a crise no estado trouxe dificuldades nos pagamentos dos adicionais de polícia e com isso fez o investimento em segurança pública cair, sobretudo nos setores de inteligência.

Para Olliveira (2017), é preciso rever a política de segurança no estado, tem que se questionar o modelo de segurança em que se tem investido, principalmente agora, onde o estado está em crise.

\subsection{MACHINE LEARNING}

A Aprendizagem de Máquina, do inglês machine learning, é utilizada na fase de mineração de dados do processo de descoberta do conhecimento em base de dados e surgiu da percepção de criar programas computacionais que aprendam um determinado comportamento ou padrão automaticamente, a partir de exemplos ou observações. A ideia por trás da aprendizagem é que percepções devem ser usadas não apenas para agir, mas também para melhorar a habilidade do agente para agir no futuro. (RUSSEL e NORVIG, 2009)

O processo de pesquisa em aprendizado de máquina consiste em examinar e experimentar as estratégias mais eficazes para a construção de programas que aprendem a partir da experiência adquirindo conhecimento de forma automática. Um programa aprende um conjunto de tarefas $\mathrm{T}$ com uma medida de desempenho $\mathrm{D}$ a partir de uma experiência $\mathrm{E}$, se seu desempenho de aprendizado D aumenta com a experiência E, ou seja, se é capaz de tomar decisões baseado em experiências acumuladas por meio da solução bem-sucedida de problemas anteriores. (MITCHELL, 1997)

\section{PROPOSTA DE SOLUÇÃO}

\subsection{A CRIMINALIDADE NO RIO DE JANEIRO}

O índice de criminalidade no Rio de Janeiro só aumenta, alguns motivos agravaram essa situação, sendo eles a deterioração das Unidades de Polícia Pacificadoras (UPPs), crise financeira no estado, expansão da mancha de criminalidade, fortalecimento de facções criminosas e a "pacificação" versus confronto.

A deterioração das UPPs é notada quando se tem o aumento de 13,746\% de números de tiroteios nos últimos anos em favelas que contém UPPs, onde o número de confrontes nessas favelas com UPPs passou de 13, em 2011, para 1.555, em 2016. Para Julita Lemgruber (2017), a aposta primordial nas UPPs foi um dos problemas, pois ela tinha apenas foco na capital e não como uma política de segurança para todo o estado, prova disso que apenas 1 das 38 UPPs implantadas fica no complexo da Mangueirinha, que fica na Baixada Fluminense. Para Ignacio Cano (2017), faltou uma “manutenção" nas UPPs, 
afirmando que os gestores se limitaram a uma quase expansão automática das UPPs.

A crise financeira no estado vem contribuindo diretamente para o aumento dessa criminalidade, policiais não vem recebendo em dia e suas condições de trabalho não são boas. Maria Isabel (2017), afirma que a escassez de recursos vem atrapalhando a infraestrutura e as condições de trabalho da polícia, aumentando assim a vulnerabilidade de policiais e das pessoas que habitam na cidade.

A expansão da mancha de criminalidade, com a instalações de UPPs nas comunidades da capital do estado do Rio de Janeiro, criminosos começaram a fugir para cidades vizinhas e assim o índice de criminalidade nessas regiões começou a aumentar. Para Couto (2017), essa expansão fez com que a UPP que era considerável uma ideia infalível se desmantelasse.

O fortalecimento de facções criminosas também tem sido um problema grave, com a crise instalada no estado, as facções vêm aproveitando a fragilidade na segurança para agir de formas mais agressivas. Para Julita Lemgruber (2017), a criminalidade nota que existe uma fragilidade na segurança pública, que não há rumo, orientação. A desigualdade social e a falta de oportunidade para jovens moradores de comunidades continuam a contribuir para esse fortalecimento, as oportunidades dadas pelo tráfico têm seduzidos jovens que não tem oportunidades do lado de fora dessas comunidades, com isso acabam entrando na vida do crime.

A pacificação versus o confronto, com a "falência” das UPPs e consequentemente o aumento da criminalidade, dados comprovam que o confronto armado está voltando a ganhar força. O aumento no número de pessoas mortas pela polícia reflete o aumento de confrontos armados. Nos últimos anos, o número de homicídios consequentes de oposição à intervenção policial teve um aumento de 120\% chegando a 920 em 2016. Com 419 mortes, em 2012 foi registrado o índice mais baixo da última década e em 2007, antes das instalações das UPPs, o número de mortos por policiais chegou a 1.330.

\subsection{CENÁRIO Do BAIRRo da TIJUCA}

O estudo de caso foi realizado no bairro da tijuca localizada na cidade do Rio de Janeiro, por ser uma das 20 áreas mais críticas da cidade do Rio de Janeiro, se tratando de segurança pública.

O bairro escolhido para o estudo detém a terceira maior variação de índices de roubo de 2016 para 2017. A tijuca tem também a maior variação de roubos a pedestres, de 2016 para 2017 o aumento foi de 38\%.

De acordo com o ISP, o total de roubos registrados nas três delegacias que abrangem o bairro da Tijuca, aumentou de 3.722 casos para 5.006, um aumento de 34,4\%. Roubos a veículos cresceram em $54,7 \%$, ao comercio de $31,7 \%$ e roubos a celulares um crescimento de $45,1 \%$.

Segundo o levantamento de dados do aplicativo Fogo Cruzado, o bairro da tijuca é o quinto com mais números de tiroteios registrados, se destacando pelo grande número de pessoas que vieram a falecer em decorrência desses tiroteios. Foram registradas 32 vítimas fatais no bairro, ficando assim em segundo lugar nas estatísticas, como pode ser verificado na Figura 3 a seguir. 
Figura 3 - Os 10 bairros com mais tiroteios no Rio

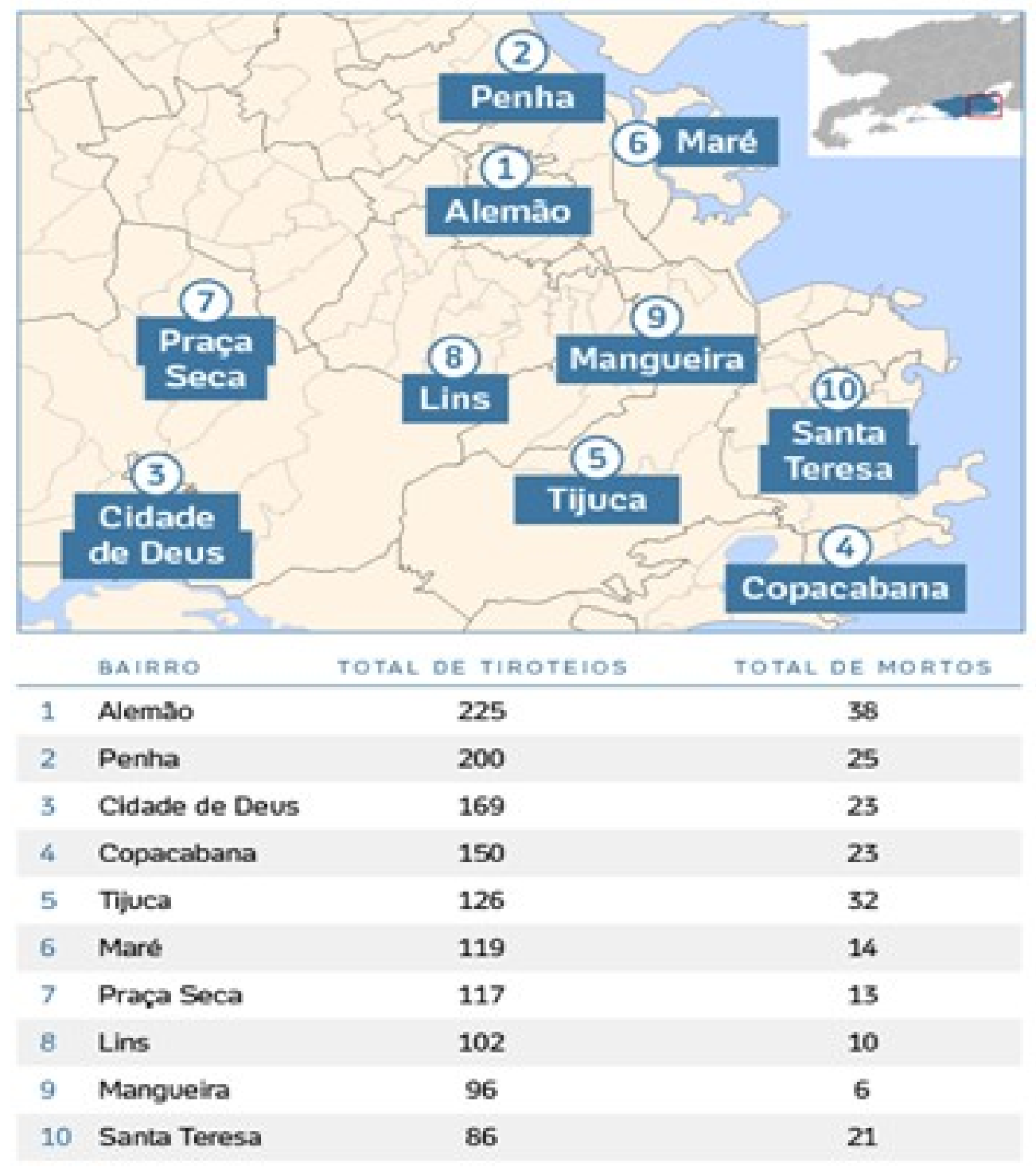

Fonte: UOL (2017)

\subsection{SITUAÇÃo DA PMERJ}

Ainda que seja uma profissão que envolva um alto risco, a cada dia que passa se causa mais surpreendente as altas taxas de vitimização de policiais militares no Rio de Janeiro. O Gráfico 1 a seguir irá demonstrar a evolução do índice de mortes de policiais nos últimos 23 anos no estado do Rio de Janeiro, poderá ser observado que nos últimos 2 anos o aumento foi consideravelmente acelerado, consequentemente o enfraquecimento das UPPs foi colocado a público. 
Gráfico 1 - Índice de mortes de policiais

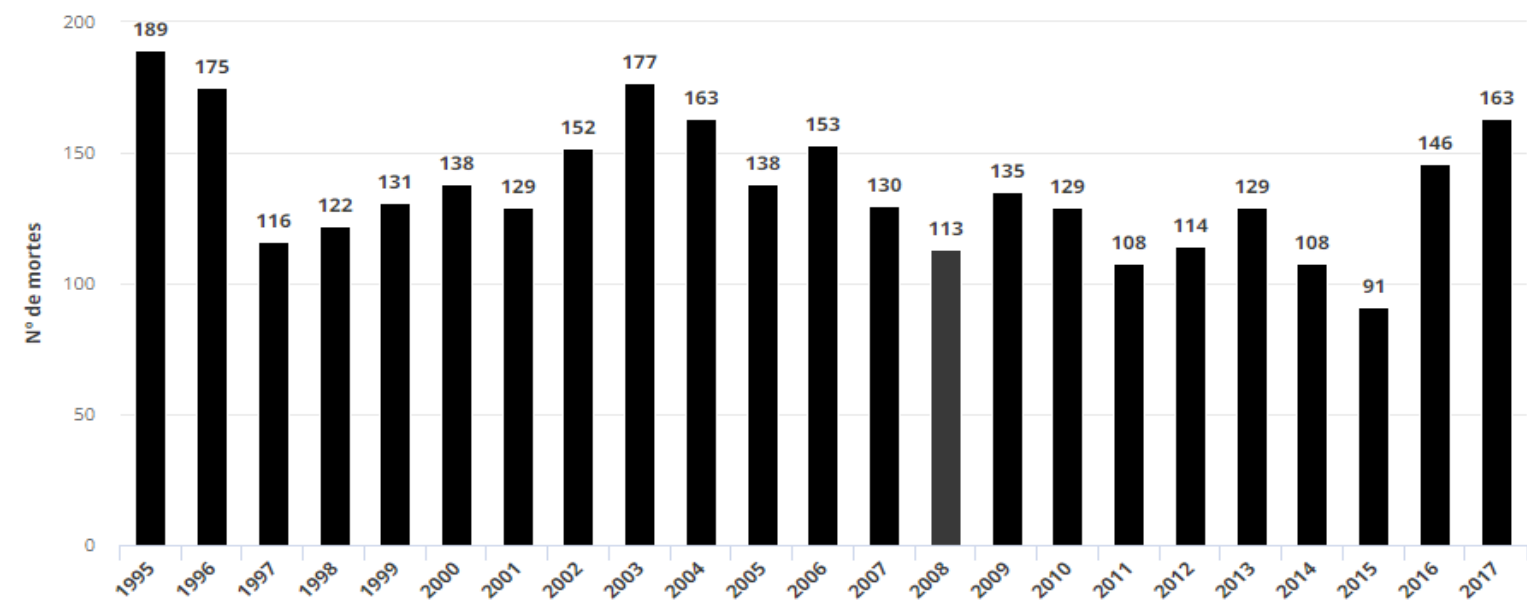

Fonte: O Globo (2018)

A perda de efetividade nas UPPs vem contribuindo para o aumento da morte de policiais a cada dia que passa com o crescimento da criminalidade e consequentemente a falta de investimento nas UPPs o confronto direto entre polícia e bandido aumentou. A Comissão de Análise de Vitimização da Polícia Militar do Rio de Janeiro fez um estudo que teve como resultado a indicação da "desmobilização" das UPPs, tendo foco em unidades que estão sendo sufocadas pelo crime. Um dos objetivos seria levar esse efetivo a ser realocado para batalhões locais.

Na Figura 4 a seguir, observam-se as 10 UPPs que mais sofrem ataques violentos.

Figura 4 - As 10 UPPs com mais ataques a policiais

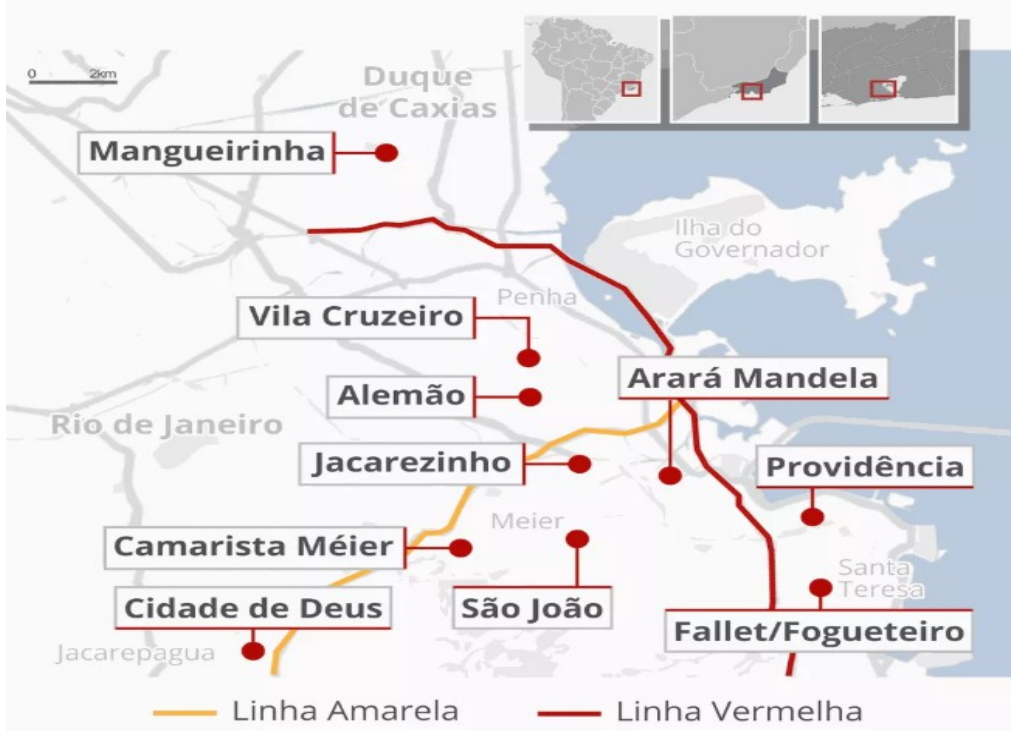

Fonte: O Globo (2018)

Segundo um levantamento do Ministério Público do Rio de Janeiro feito no final de 2017, o latrocínio ou provável latrocínio, são os maiores responsáveis pela causa de morte no Rio de Janeiro. De acordo com esse levantamento dos 163 mortos em 2017, sendo que 128 se encontravam de folga e 35 estavam em serviço. Para Ubiratan Ângelo, ex-comandante da Polícia Militar, muitos casos os policiais estão armados na folga e acabam reagindo de forma equivocada. Uma prova disso é que policiais das Forças Especiais não estão tão presentes entre esses números. 
As regiões que mais se destacam entre esses números são, Complexos da Maré, Jacarezinho e bairros da Zona Norte da cidade do Rio de Janeiro, já na morte por latrocínio uma das principais causas de morte de policial na cidade, Figura 5, o município de São Gonçalo é o de maior destaque.

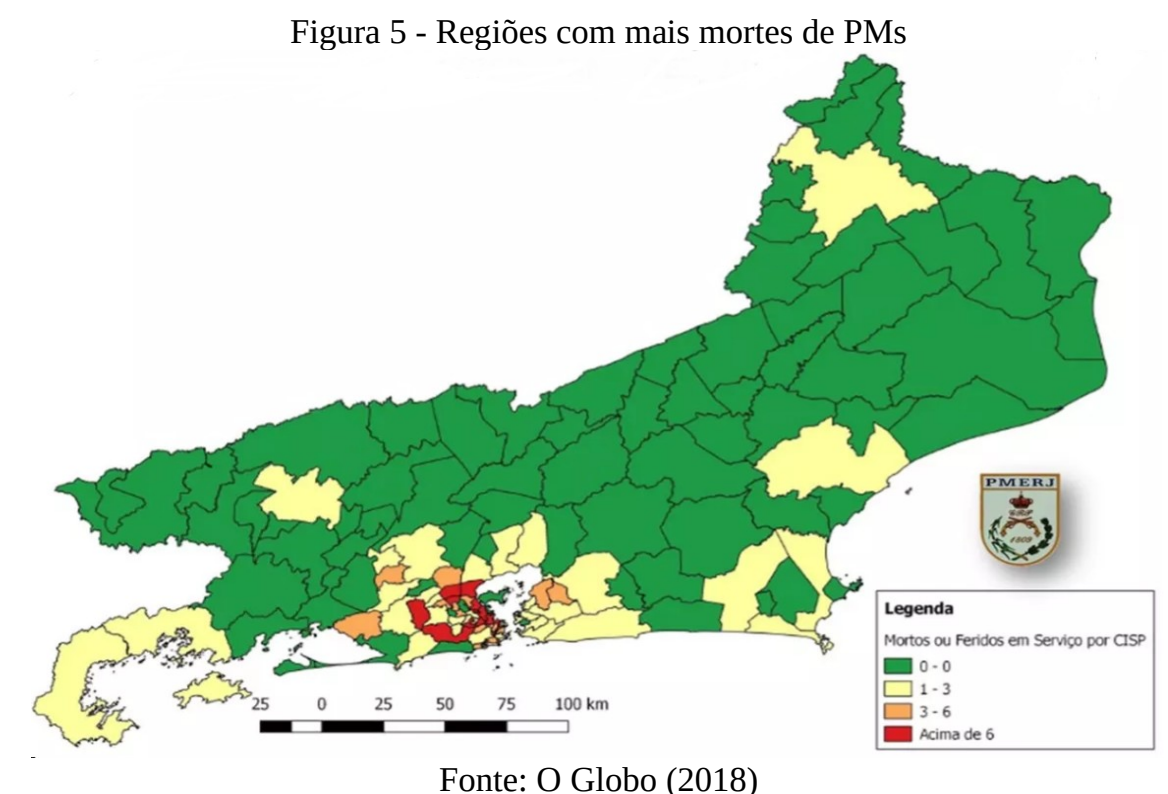

\subsection{UTILIZAÇÃO DA INTELIGÊNCIA ARTIFICIAL}

Com a utilização da inteligência artificial, a expectativa é que o sistema consiga incorporar registros de sexo e idade das pessoas envolvidas, como esses crimes foram cometidos. Uma variável que também será levada em conta será é o tempo e os dados sobre o terreno e tudo que há em volta de onde ocorreu o crime.

Em New Orleans, a prefeitura da cidade alimentou um software, como todo o arquivo do departamento da polícia da cidade que consequentemente foram cruzados com informações públicas das redes sociais e mapas da cidade. A empresa que prestou o serviço conseguiu identificar que havia como descobrir conexão de certas pessoas com gangues locais, ou a chance de certas pessoas cometerem crimes.

Através do uso dessa tecnologia é possível identificar os possíveis autores de um crime a partir de informações obtidas pela polícia. O número de assassinatos em New Orleans obteve uma redução de $25 \%$ nos três anos consecutivos a instalação dessa tecnologia, o que indica uma eficácia do sistema.

No Japão, a polícia municipal da cidade de Kanagawa, criou um sistema que é capaz de prever quando e onde crimes e acidentes têm mais chances de ocorrer e deverá ser colocado em prática já nos próximos anos. A tecnologia irá utilizada a inteligência artificial para analisar dados de aproximadamente 1 milhão de crimes que já ocorreram. Hoje os policiais já utilizam outro sistema que mostra áreas e períodos do dia em que os crimes mais ocorrem para poder melhor assim criar uma logística para as patrulhas.

No Paraná, o clube de futebol Atlético Paranaense vem utilizando a biometria para capturar criminosos que estão em dívida com a justiça. Existe um banco de dados que vem sendo interligado entre clube e o poder público, como cerca de dois terços dos seus mais de 11 milhões de habitantes já fizeram o cadastramento biométrico, foi só o clube fazer um convenio junto ao TJ-PR, DETRAN-PR, Secretaria de Segurança Pública e Celepar (Companhia de Tecnologia da Informação e Comunicação do Paraná), o sistema do estádio do clube está conectado a essa base digital. 


\subsection{APLICAÇÃo MACHINE LEARNING}

Com o aumento da criminalidade é essencial que todas as secretarias e demais departamentos do estado consigam está um passo à frente do crime e que consigam fazer o mapeamento correto de todas as informações para que todas as medidas preventivas tenham o máximo de assertividade possível.

Essa ferramenta poderá ser usada para identificar padrões que são ocultos em meio a milhões de registros de policiais e através da análise preditiva, determinando assim a probabilidade de onde irão ocorrer novos crimes, as informações podem ser disponibilizadas em um mapa de calor atualizado em tempo real garantindo assim uma visão dinâmica e altamente precisa sobre a criminalidade na sua jurisdição. Com essas informações poderá ser feita uma melhor logística para o patrulhamento de viaturas da polícia em horários que foram identificados como críticos.

É possível posicionar agentes estrategicamente para inibir crimes antes mesmo que eles aconteçam otimizando assim a vigilância, montar estratégias utilizando filtros para encontrar insights específicos como quarteirões com maiores riscos de ocorrências durante a tarde, ou algumas rodovias sujeitas a roubo de carga. À medida que novos dados são inseridos, a ferramenta se adapta para acompanhar esses novos padrões de ocorrência, atualizando assim o mapeamento em tempo real.

Além da análise de crimes passados, é possível também fazer a previsão de eventos futuros, sendo assim uma análise preditiva precisa. Essa tecnologia também é capaz de realizar análises prescritivas, indicando assim os melhores cursos de ação e os possíveis resultados para a tomada de decisão.

\section{CONSIDERAÇÕES FINAIS}

O presente trabalho conseguiu atingir o seu objetivo geral de propor melhorias no processo de combate à criminalidade na cidade do Rio de Janeiro, ao empregar o big data para coletar as informações e contribuir para a tomada de decisão, a fim de melhor gerenciar as informações geradas a cada crime ocorrido.

Foi identificado o aumento acelerado da criminalidade no bairro da Tijuca, onde foi proposto o uso da tecnologia de machine learning, como um redesenho na inteligência da segurança pública para poder combater esse aumento da criminalidade.

A proposta apresentada, através de um novo redesenho, envolve melhorias no setor de inteligência, sendo assim a aplicação de novo procedimento onde as informações foram levantadas através de registros de crimes na cidade do Rio de Janeiro.

Após apresentado às propostas e as melhorias necessárias, foram descritos benefícios da aplicação do machine learning. Onde pode se destacar, atuação preventiva, otimização da vigilância, precisão e previsão dos crimes. Contudo, a aplicação da tecnologia deve ser monitorada, para poder saber se de fato ela traz benefícios para o combate à criminalidade.

\section{REFERÊNCIAS BIBLIOGRÁFICAS}

ARTHUR, Trindade. Estatística do caos: Violência no Rio é a que mais aumenta.

Disponível em: https:/www.gazetaonline.com.br/noticias/policia/2018/02/estatistica-docaos-violencia-no-rio-e-a-que-mais-aumenta-1014120694.html. Acesso em: 15/09/2018.

BEATH, C. et al. Finding value in the information explosion. MIT Sloan Management Review, v. 53, n.4, 2012. 
CHOO, Chun Wei. A organização do conhecimento: como as organizações usam a informação para criar significado, construir conhecimento e tomar decisões. 2 ed. São Paulo: SENAC São Paulo, 2006.

DAVENPORT, T.; BARTH, P.; BEAN, R. How 'Big data' is different. MIT Sloan Management Review, July 2012.

DEMIRKAN; et al. Leveraging the capabilities of service-oriented decision support systems: Puttinganalytic and big data in cloud. Decision support system, 2012.

GALBRAITH, JR. ORGANIZATION DESIGN CHALLENGES RESULTING FROM BIG DATA. Journal of Organization Design. 3, 1, 2-13, jan. 2014. ISSN: 2245408X.

GOMES, L. F. A. M. Teoria da Decisão. São Paulo: Thomson Learning, 2007.

GRANTZ, J.; REINSEL, D. The digital universe in 2020: Big data, bigger digital shadows, and biggest growth in the far east. IDC iView: IDC Analyze the Future, n. v.2007, p. 1-16, 2012.

HAMMOND, Kristian. The Value of Big Data isn't the Data. Blog da Harvard Business Review, May 2013. Disponível em: << https://blogs.hbr.org/cs/2013/05/the-value-of-bigdata-isnt-the.html $>>$ Acesso em: 11/09/2018.

HARRIOTT, Jesse. 7 Pillars for Successful Analytics Implementation. Marketing Insights, spring, 2013.

IGNACIO, Cano. Estatística do caos: Violência no Rio é a que mais aumenta.

27/02/2018. Disponível em:

https://www.gazetaonline.com.br/noticias/policia/2018/02/estatistica-do-caos-violencia-norio-e-a-que-mais-aumenta-1014120694.html. Acesso em: 15/09/2018.

MANYIKA, James; et al. Big Data: The next frontier for innovation, competition, and productivity. Mckinsey Global Institute, maio, 2011; Disponível em:

$<$ Http://wwwmckinsey.com/insights/mgi/research/technology_and_innovation/

bid_data_the_next_frontieer_for_innovation> Acesso em: 20/09/2018.

MCAFEE, Andrew; BRYNJOLFSSON, Erik. Big Data: The Management Revolution. Harvard Business Review, outubro, 2012.

MITCHELL, T., Machine learning. New York: McGraw-Hill, 1997.

O GLOBO. Mortes por intervenção policial no Rio. (2017) Globo. Disponível em: < https://g1.globo.com/rio-de-janeiro/noticia/mortes-por-intervencao-policial-no-rj-seaproximam-de-patamar-de-antes-das-upps.ghtml> Acesso em: 15/09/2018

OLIVEIRA, Cecilia. Do Alemão a Copacabana: Ranking revela os 10 bairros com mais tiroteios do Rio. Disponível em: https://noticias.uol.com.br/cotidiano/ultimas-noticias/2017/ 08/08/do-alemao-a-copacabana-ranking-mapeia-bairros-com-maior-numero-de-tiroteios-norio.html. Acesso em: 15/09/2018.

PHELAN, Mike. The Death Of Big Data. Site da Forbes, 10 abril 2012. Disponível em: <http://www.forbes.com/sites/ciocentral/2012/10/04/the-death-of-big-data/2/:> Acesso em: $21 / 09 / 2018$

PREIMESBERGER, Chris. Big Ideas about Big Data. EWeak, 15 agosto. 2011.

RUSSELL, S., NORVIG, P., Artificial Intelligence: A Modern Approach, Prentice-Hall, Englewood Cliffs, NJ, 2009. 
TANKARD, Colin. Big Data Security. Network security, jul. 2012.

TAURION, Cezar. Você realmente sabe o que é big data? Blog da IBM, 30 abril 2012. Disponívelem:<https:/www.ibm.com/developerworks/blogs/ctaurion/entry/ voce_realmente_sabe_o_que_e_big_data?lang=em. Acesso em: 20/09/2018>

WEBER, K. et. al. One size does not fit all - a contigency approach to data governance. ACM J. Data Inform. Quality 1,1 Article 4, junho 2009, 27 p 